\title{
Microwave study of screened two-dimensional electron crystals on helium films
}

\author{
G. Mistura \\ Fakultät für Physik, Universität Konstanz, D-78434 Konstanz, Germany \\ and Grenoble High Magnetic Field Laboratory, \\ Max-Plank Institut für Festköperforschung/Centre National de la Recherche Scientifique, Bôite Postal 166, \\ F-38042, Grenoble, Cedex 9, France \\ T. Günzler, S. Neser, and P. Leiderer \\ Fakultät für Physik, Universität Konstanz, D-78434, Konstanz, Germany
}

(Received 6 December 1996)

\begin{abstract}
We report investigations at microwave frequencies on surface-state electrons (SSE) which were supported by helium films adsorbed on different polymer substrates at temperatures above $1 \mathrm{~K}$. The real and imaginary parts of the dielectric response $\epsilon(k, \omega)$ of the SSE were measured at fixed $k$ and $\omega$ inside a $10 \mathrm{GHz}$ microwave cavity. The solidification of the two-dimensional electron crystal was detected by a kink occurring simultaneously in both components of $\epsilon(k, \omega)$ as the electron density was slowly increased at constant temperature. The influence of the dielectric substrate on such a freezing transition has been measured at different temperatures and at various helium-film thicknesses. The results are found to be in good agreement with existing theories. This work represents the first systematic study of a screened two-dimensional electron crystal on helium.
\end{abstract}

\section{INTRODUCTION}

For the past two decades, surface-state electrons (SSE's) on a liquid-helium surface have been the object of an intense experimental and theoretical investigation because they form a particularly clean realization of a two-dimensional electron system (2DES). ${ }^{1}$ The surface of liquid helium is an almost ideal substrate for the SSE's owing to its pure and predictable experimental conditions. Furthermore, the electronic areal density $n$ and the coupling of SSE's to the surface can be easily changed over a relatively wide range of values. Thus SSE's on liquid helium have been a perfect testing ground on which to test the effect of the Coulomb interaction on the transport and equilibrium properties of a 2DES. A large number of interesting phenomena have been found, the most dramatic perhaps being the detection of a Coulomb crystal phase $^{2}$ and, more recently, the possible observation of the melting of this solid due to zero point energy fluctuations ${ }^{3}$ (quantum melting), a phase transition predicted long time ago by Wigner. ${ }^{4}$

On bulk liquid helium $n$ is limited to a value $\leqslant 2.2 \times 10^{9} \mathrm{~cm}^{-2}$ due to an electrohydrodynamic instability of the charged surface. ${ }^{1}$ At this density a Coulomb crystal forms at a temperature below $1.05 \mathrm{~K},{ }^{2,5}$ while the Fermi energy of the 2DES, $T_{F}=\pi \hbar^{2} n / k_{B} m$, where $m$ is the electron mass, is equal to $61 \mathrm{mK}$. Thus electrons on bulk helium always form a classical system in contrast to 2DES in semiconductor inversion layers and quantum well structures where they are always in a degenerate state. ${ }^{6}$ In order to bridge the gap between these two different regimes, it has been suggested to use a thin helium film as a substrate for the SSE's, because a film is additionally stabilized by van der Waals forces. ${ }^{7}$ This has been demonstrated by Etz et al. ${ }^{8}$ who were able to charge helium films to electron densities up to $\simeq 10^{11} \mathrm{~cm}^{-2}$.
At these densities, the initial film thickness is greatly reduced and is essentially determined by the large electrostatic pressure ${ }^{8} n^{2} e^{2} / 2 \epsilon_{0}$, $e$ being the electron charge. Thus, in contrast to the pure conditions on bulk helium, SSE's on a thin helium film are perturbed by the roughness of the underlying substrate and by the presence of surface impurities, which lead to a progressive disappearance of the signal in low-frequency conductivity measurements as $n$ approaches $10^{11} \mathrm{~cm}^{-2}$. To overcome such a limitation we have employed a microwave cavity technique (resonance frequency $f \simeq 10 \mathrm{GHz}$ ). This is quite a sensitive technique because it probes the motion of the SSE's over very small horizontal distances, $\approx 10 \AA$ in our case (see Sec. III), so reducing the influence of surface defects on the electronic motion.

This paper is organized as follows. After a brief discussion of the theoretical phase diagram of SSE's on a helium film, we will describe our microwave cavity technique, focusing particularly on the rather stringent specifications that the support of the helium film, inserted along the cavity axis, has to satisfy. We then illustrate the formulas used in our data analysis, before presenting our experimental results together with a phenomenological model which explains them.

\section{THE SCREENED PHASE DIAGRAM}

The phase diagram of a 2DES system on bulk liquid helium was calculated within a mean-field approximation by Platzmann and Fukuyama. ${ }^{9}$ In the classical low-density and high-temperature region, the shape of the gas-solid boundary line can be easily determined by a simple energetic argument. It is convenient to introduce the plasma parameter $\Gamma=\langle V\rangle /\langle K\rangle$, where $\langle V\rangle$ and $\langle K\rangle$ are the mean potential and mean kinetic energies per electron, respectively. Generally a large potential energy favors crystallization. For a 2DES, $\langle V\rangle=\left(e^{2} / 4 \pi \epsilon_{0} \bar{\epsilon}_{\mathrm{He}}\right) \sqrt{\pi n}$, where $\bar{\epsilon}_{\mathrm{He}}=\left(\epsilon_{\mathrm{He}}+1\right) / 2, \epsilon_{\mathrm{He}}$ being 
the dielectric constant of liquid helium, while, in the classical region, $\langle K\rangle=k_{B} T$. Thus the freezing boundary line satisfies the simple relation $k_{B} T_{m}=e^{2} \sqrt{\pi n} / 4 \pi \epsilon_{0} \bar{\epsilon}_{\mathrm{He}} \Gamma$, where $\Gamma \approx 127$ from experiments ${ }^{2,5}$ while the theoretical value ${ }^{10}$ based on the Kosterlitz-Thouless melting criterion is $\Gamma \approx 125$.

On a thin helium film the interparticle interaction is modified due to the screening of the electron-electron interaction by the substrate. For small interparticle distances, $(\pi n)^{-1 / 2} \ll d$, where $d$ is the helium film thickness, the screening is negligible and one has a Coulombic potential $V(r) \sim e^{2} / 4 \pi \epsilon_{0} \bar{\epsilon}_{\mathrm{He}} r$. If instead the electrons are far apart, $(\pi n)^{-1 / 2} \rightarrow d$, we have ${ }^{11} \quad V(r) \sim e^{2}(1-\delta) / 4 \pi \epsilon_{0} \bar{\epsilon}_{\mathrm{He}} r$ $+2 \delta e^{2} d^{2} / 4 \pi \epsilon_{0} \bar{\epsilon}_{\mathrm{He}} r^{3}, \quad$ with $\delta=\left(\epsilon_{\mathrm{sub}}-1\right) /\left(\epsilon_{\mathrm{sub}}+1\right), \quad \epsilon_{\mathrm{sub}}$ being the dielectric constant of the substrate underneath the helium film.

The major physical consequence of using the helium film on a dielectric substrate, the case of our experiment, is simply the renormalization of the electron charge and the shrinking of the solid phase region with respect to the 2DES phase diagram on bulk helium. ${ }^{11}$ The classical freezing line is pushed to higher density and lower temperature, while the quantum (Wigner) melting is shifted to lower $n$ and $T$. Such a shrinking of the solid phase becomes more pronounced on substrates with large $\epsilon$. The theoretical expression for the classical freezing line based on the Kosterlitz-Thouless melting criterion has been given by Saitoh as ${ }^{12}$

$$
k_{B} T_{m}(d)=\frac{e^{2} \sqrt{\pi n}}{4 \pi \epsilon_{0} \bar{\epsilon}_{\mathrm{He}} \Gamma}\left[1-\frac{\delta}{\left(1+4 \pi n d_{\mathrm{He}}^{2} / c^{2}\right)^{3 / 2}}\right],
$$

where $c=1.1061$ and $d_{\mathrm{He}}$ is the helium film thickness. It may be worthwile to point out that in a real experiment, in contrast to the mentioned theoretical analyses, ${ }^{11,12} d_{\mathrm{He}}$ is not a completely independent parameter due to the thinning effect of the helium film caused by the SSE's electrostatic pressure. Actually, its relation to the uncharged film thickness $d_{0}$ is ${ }^{8}$

$$
d_{\mathrm{He}}(n)=\frac{d_{0}}{\left(1+n^{2} e^{2} / 2 \epsilon_{0} \rho_{\mathrm{He}} g H\right)^{1 / 3}},
$$

with $\rho_{\mathrm{He}}=145 \mathrm{~kg} / \mathrm{m}^{3}$ the density of liquid helium, $g$ the acceleration of gravity, and $H$ the height of the substrate with respect to the liquid helium surface. As an example, for $n=5 \times 10^{10} \mathrm{~cm}^{-2}, d \approx 96 \AA$ when $d_{0}=200 \AA$ and $d \approx 99 \AA$ when $d_{0}=400 \AA$.

\section{EXPERIMENTAL SETUP}

In our experiment we have used a cylindrical microwave cavity, $19.5 \mathrm{~mm}$ in diameter and $20 \mathrm{~mm}$ in length, which was excited in the fundamental $\mathrm{TM}_{010}$ mode. When the Si platelet was inserted along the axis of the cavity (see Fig. 1) the resonance frequency was $f_{0} \approx 10 \mathrm{GHz}$. The resonator was mounted in a microwave transmission circuit. The output power of the $X$-band generator HP 8206 was attenuated by a variable cursor attenuator before being fed into the cavity (typical attenuation $\approx-40 \mathrm{~dB}$ ) via a special lowtemperature, low-loss microwave coaxial cable. The transmitted power through the cavity was amplified by a pair of high-stability, low-noise $X$-band MITEQ amplifiers (total gain of $36 \mathrm{~dB}$ ). A feedback circuit kept the microwave generator phase locked to the cavity resonance and both reso-

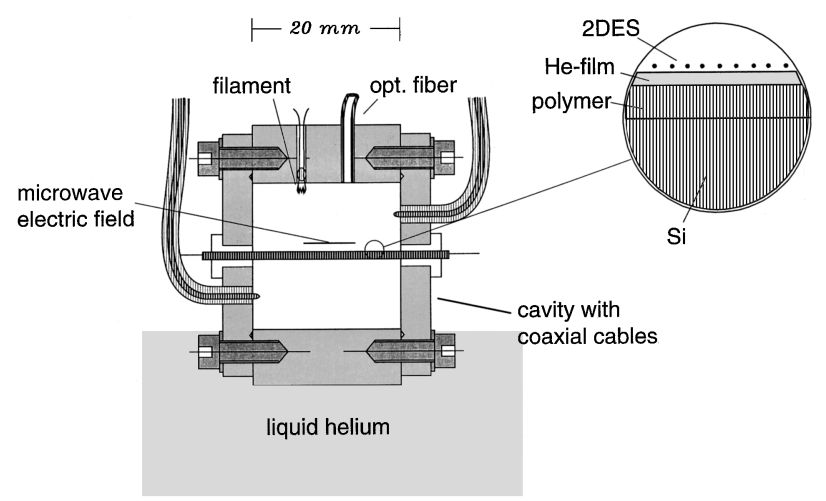

FIG. 1. Schematic drawing of the microwave cavity used in the experiment. The blow up shows the typical layered structure of the SSE support.

nance frequency and transmitted power through the cavity were measured and stored in a personal computer. This represents an important experimental improvement over previous studies, where the microwave absorption of the 2DES was monitored at a constant frequency. ${ }^{13,14}$

The resonator was weakly coupled and the geometry was thus free from coupling effects through temperature variation. The input microwave power amounted to less than 500 $\mathrm{nW}$, including the attenuation due to the coupling, and the low-temperature quality factor with no electrons was $Q_{0} \simeq 3000$. This gives a maximum value for the microwave electric field amplitude $E_{0} \simeq 20 \mathrm{~V} / \mathrm{m} .{ }^{15}$ Thus the maximum electron displacement induced by $E_{0}$ can be estimated by the relation $x_{0}=e E_{0} /\left(m_{e} \omega^{2}\right) \approx 10 \AA$.

A rectangular $(\approx 30 \times 8 \mathrm{~mm}), p$-doped silicon platelet served as a mechanical support for the helium film and was placed along the axis of the cavity, parallel to the microwave electric field of the $\mathrm{TM}_{010}$ mode. Although the doping of our silicon was low (typical resistivity at room temperature $\rho \approx 20 \Omega \mathrm{cm})$ and the platelet thus was highly resistive at low temperatures, it was still conductive enough to serve at the same time as a lower electrode to hold the electrons on the film (in some cases, we had to increase its conductivity by electron-hole pairs generated by illumination with an optical fiber). A clamping voltage $U$ was applied between the platelet and the resonator housing, the latter being electrically connected to ground.

The cavity was inserted in a closed copper cell immersed in the liquid helium of a glass Dewar. Before the measurements high-purity helium gas was condensed into this sample cell at $1.2 \mathrm{~K}$. The level of liquid helium inside the cavity was precisely adjusted below the top face of the Si platelet, using the frequency shift of the cavity itself as a level meter; the frequency change due to the complete filling of the cavity by liquid helium amounted to $\approx 120 \mathrm{MHz}$. By adjusting the height $H$ between the substrate and the liquid helium level it was possible to vary the thickness of the uncharged helium film $d_{0}$ in a wide range $(\approx 300-900 \AA)$. Its indicative value was simply determined by the nonretarded ${ }^{16}$ Frenkel-Halsey-Hill relation $d_{0}=\left(\alpha / \rho_{\mathrm{He}} g H\right)^{1 / 3}$, where $\alpha$ is the van der Waals constant of the outer surface layer. For the PMMA [poly(methylmethacrylate)] layer, which will be discussed below, we have used the value appropriate for glass, ${ }^{17} \alpha \approx 26 \mathrm{~K}$ layers ${ }^{3}$. We 
estimate that the uncertainty on our determination of $d_{0}$ amounts to about $20 \%$.

For a successful experiment, the quality of the outer surface of the substrate is of paramount importance, particularly for very small $d_{0}$ and/or large electron densities $\left(\geqslant 2 \times 10^{10} \mathrm{~cm}^{-2}\right)$. If the surface is not very smooth, electrons can easily tunnel through the helium film at some rough spots where the equilibrium film thickness of the helium film $d_{\mathrm{He}}$, is substantially reduced. In the case of a conducting substrate, this causes a continuous loss of electrons which may severely limit the maximum achievable stable density of the 2 DES. ${ }^{8}$ To reduce this problem, an insulating surface can be used: its rough spots are charged by the first electrons which tunnel through the helium film and this effectively prevents further electrons from tunneling.

We have thus decided to use as a substrate for the helium film a Si platelet covered with an insulating polymer layer of thickness $d_{\text {ins }}$ with dielectric constant $\epsilon_{\text {ins }}$. For the choice of such a polymer, many different factors have to be considered. First of all, it must not alter the physical properties (mainly the microwave absorption and the dc conductivity) of the underlying $\mathrm{Si}$ platelet, and it must be mechanically stable to sustain the severe mechanical stresses which occur during a cool down. Furthermore, to avoid using very large clamping voltages, ${ }^{13,18}$ its thickness $d_{\text {ins }}$ has to be relatively thin $\left(d_{\text {ins }} \approx 200-500 \mathrm{~nm}\right)$ and, in order to guarantee a uniform $U$ (e.g., uniform $n), d_{\text {ins }}$ must be as uniform as possible. Finally, its outer surface must be very smooth so as to reduce the number of the various rough spots which may act as strong scattering centers for the 2DES in particular when electrons are attracted and stuck to them.

During the course of this experiment we have tried various insulating polymers with different results. In the beginning we tightly wrapped a Mylar foil $\left(d_{\text {ins }}=5-10 \mu \mathrm{m}\right.$, $\left.\epsilon_{\text {ins }}=3.7\right)$ around the $\mathrm{Si}$ platelet. Although the preparation was relatively simple, it was very difficult to get reproducible results. The main problem was due to the static charging of the foil induced during its handling. Furthermore, $U$ was not very well defined because of the inevitable wrinkles in the foil when it was cooled down. To avoid such a problem, we used a perflourinated-poly-ether-oil (PFPE), a highly viscous polymer oil with a very low permittivity, $\epsilon_{\text {ins }}=1.76$. We had to manually spread it over the Si platelet by means of a sharp glass edge because there is no known solvent for this oil. The resulting layer was fairly homogeneous, although this "mechanical deposition" only allowed to make relatively thick layers $\left(d_{\text {ins }} \geqslant 3 \mu \mathrm{m}\right)$. We then used PMMA $\left(\epsilon_{\text {ins }}=2.2\right)$ spin coated over a large round piece of $\mathrm{Si}$, whose central part, opportunely cut, was subsequently used as the helium film support inside the cavity. The spin coating guaranteed a thin $\left(d_{\text {ins }}: 50-300 \mathrm{~nm}\right)$, homogeneous, smooth and easily controllable insulating layer. In some experiments, we also used $\mathrm{Si}$ platelets covered by the more common $\mathrm{SiO}_{2}$. In this paper, however, we only present data which were taken on PFPE and on PMMA layers, while the data on $\mathrm{SiO}_{2}$ will be published elsewhere. ${ }^{3,19}$

The helium film was charged by firing a small filament mounted at the top of the cavity every $\approx 3 \mathrm{sec}$ and increasing at the same time the clamping voltage gradually step by step in order to guarantee a saturated charging of the film. The method ensures that the energy of the electrons is always

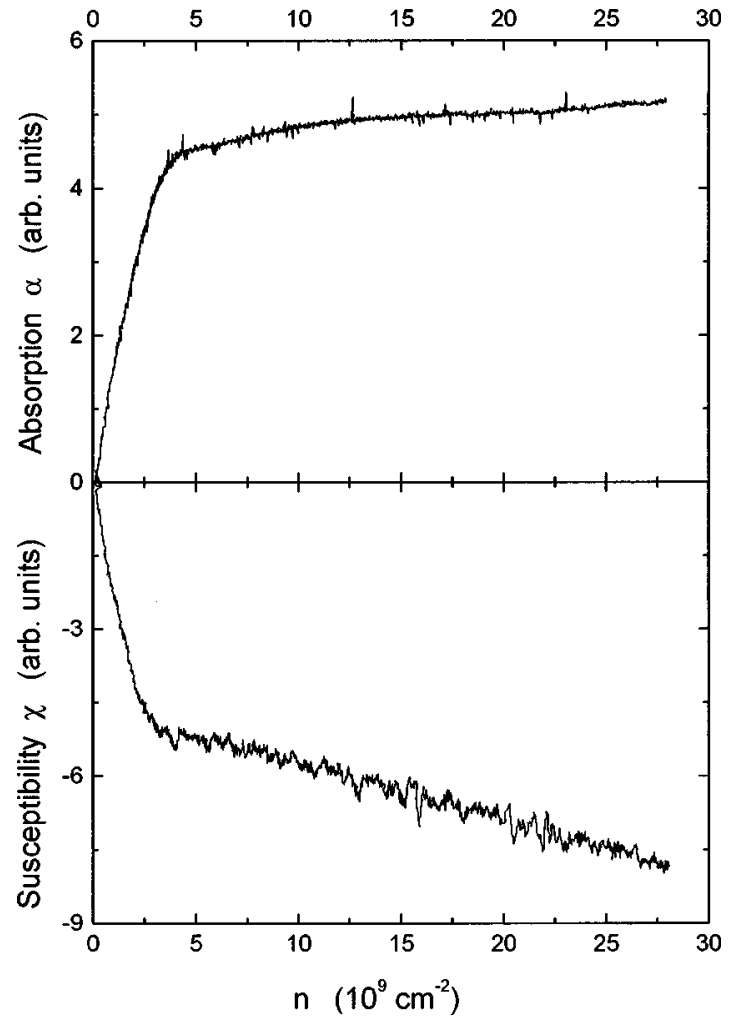

FIG. 2. Relative absorption $\alpha$ and susceptibility $\chi$ of the SSE's on a helium film adsorbed on PFPE oil in terms of electron density $n$ and clamping voltage $U$ at constant temperature $(T=1.28 \mathrm{~K})$. Initial helium film thickness is $300 \AA$.

lower than is needed to penetrate the helium. At each step the electron density $n$ was determined by

$$
n=\frac{\epsilon_{0} \epsilon_{\mathrm{ins}} \epsilon_{\mathrm{He}}}{e\left(\epsilon_{\mathrm{He}} d_{\mathrm{ins}}+\epsilon_{\mathrm{ins}} d_{\mathrm{He}}\right)} U
$$

where $d_{\mathrm{He}}$ is the equilibrium thickness of the charged helium film calculated in a self-consistent way via Eq. (2). It is evident that on an insulating layer with large $\epsilon_{\text {ins }}$ and small $d_{\text {ins }}$ high electron densities can be reached at moderate clamping voltages. On the other hand, a large $\epsilon_{\text {ins }}$ means a stronger image field for the SSE's and therefore a smaller equilibrium vertical distance from the substrate. ${ }^{20}$ Thus on a highly polarizable substrate, the SSE's are more influenced by the roughness and other defects of the surface.

\section{EXPERIMENTAL RESULTS AND DISCUSSION}

Figure 2 shows the absorption $\alpha$ and the susceptiblity $\chi$ of SSE's on a helium film $\approx 300 \AA$ thick, covering a PFPE oil $\approx 3,5 \mu \mathrm{m}$ thick, as functions of the electron density $n$ (the density values have an uncertainty of about $25 \%$, mainly due to the uncertainty in the determination of the PFPE thickness). The dimensionless relative absorption $\alpha$ was calculated using

$$
\alpha=\frac{1}{Q_{0}}\left(\sqrt{\frac{P_{0}}{P}}-1\right),
$$

where $P$ and $P_{0}$ are the transmitted microwave powers with and without electrons, respectively, while the effective sus- 
ceptibility $\chi$ of the SSE's was determined from the resonance frequency $f$ during charging with respect to the bare resonance frequency $f_{0}$ via

$$
\chi=\frac{c^{2} k_{\mathrm{res}}^{2}}{(2 \pi)^{2}}\left(\frac{1}{f^{2}}-\frac{1}{f_{0}^{2}}\right),
$$

where $c$ is the speed of light and $k_{\text {res }}=2.405 / a, a$ being the cavity radius, is the fundamental wave number of the resonator. The quantities $\alpha$ and $\chi$ can be regarded as relative measures for the imaginary and real components of the dielectric function $\epsilon(\omega, k)$ of the two-dimensional electron plasma at fixed $\omega$ and $k$. (The value of the wave vector $k$ is related to the dimensions of the boundaries of the plasma: for longitudinal plasma excitation the length of the cavity, 20 $\mathrm{mm}$, and for transversal excitation the width of the platelet, $\approx 8 \mathrm{~mm}$ ).

On charging the film the absorption rises almost linearly with increasing electron density (Fig. 2). The susceptibility is also linear and has a negative value which by comparison with Eq. (5) means that the charging has led to an increase of the cavity resonance. This is the familiar behavior of free, noninteracting electrons obeying the Drude model where both the absorbed power and susceptibility are simply linear in $n$. Generally, the sign of the susceptibility indicates whether the SSE response is in phase (plus) or out of phase (minus) with the driving force. In the low-density region of Fig. 2 we can therefore identify our SSE system as an approximation of an infinitely extended sheet of free electrons with a characteristic frequency tending to zero. Applying the Drude theory to this initial part we can derive a value for the SSE mean momentum relaxation time $\tau \approx 2.5-3 \times 10^{-11} \mathrm{~s}$ using $\tau=-A(\chi / 2 \pi f \alpha)$, where $A \approx 1.25$ is a geometrical factor. ${ }^{15}$ This value for $\tau$ is only about $\approx 2.5-3$ times smaller than the value on bulk helium calculated at the same temperature. ${ }^{21}$ Thus, the surface roughness of these low polarizable polymer substrates does not seem to affect too much the mobility of the SSE's. On the other hand, highfrequency measurements carried out at $9.4 \mathrm{GHz}$ by the Kharkov group ${ }^{13}$ on helium films wetting a pyroceramic substrate show an exponential decrease in the mobility by a factor $\approx 60$ as $d_{0}$ decreases from 80 to $25 \mathrm{~nm}$. In contrast, Jiang, Stan, and Dahm ${ }^{22}$ have measured low-frequency mobility values on helium films covering a smooth glass surface which are smaller than theoretical calculations for bulk helium only by a factor $\approx 5$ even on films as thin as $22 \mathrm{~nm}$. Other published results ${ }^{1,23,24}$ taken on different substrates wetted by helium films of comparable thickness and at similar temperatures indicate a very broad range of mobility values. In other words, the quality of the surface is of paramount importance to achieve high SSE mobility on films.

As $n$ increases, the SSE's remain in a fluid phase characterized by an increasing electronic correlation. At $n \simeq 3.5 \times 10^{9} \mathrm{~cm}^{-2}$, there is a pronounced kink in both $\alpha$ and $\chi$ which signals a change in the SSE mobility. We identify this kink as the onset of Wigner crystallization. More precisely, the change in the electron absorption at solidification is due to the pinning of the 2DES crystal to some surface defects. In reality, as the 2DES experiences a strong random potential due to surface roughness and pinned electrons, it is more likely that the 2DES freezes in many small crystallites

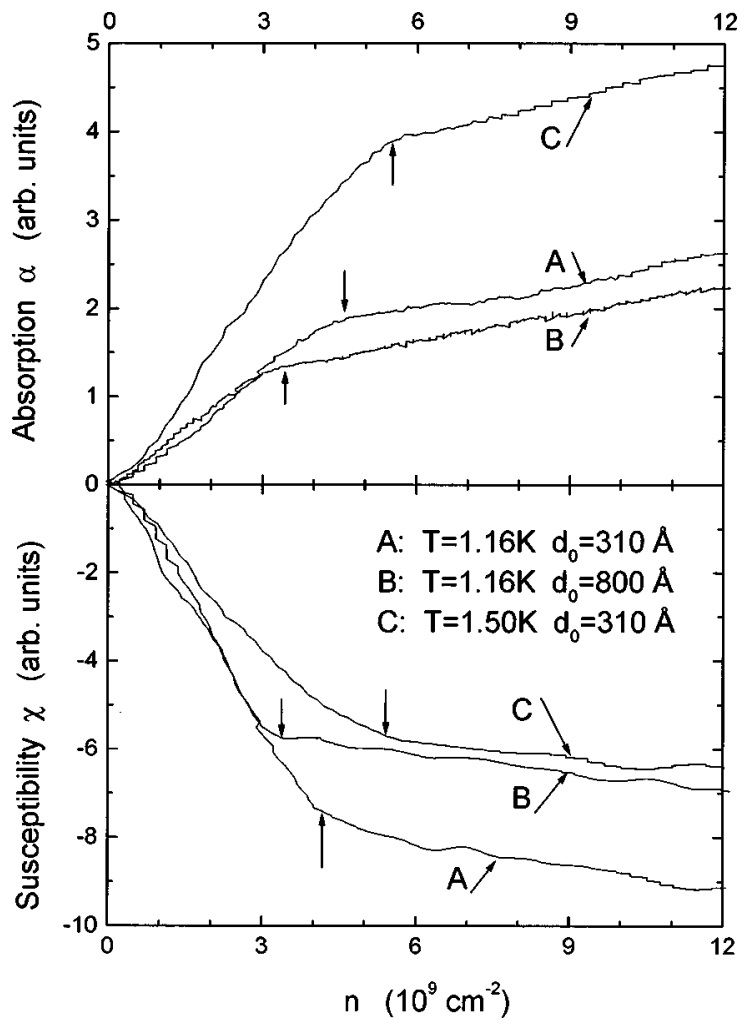

FIG. 3. Relative absorption $\alpha$ and susceptibility $\chi$ of the SSE's at two different temperatures and on two helium films of different initial thickness covering a PMMA layer versus electron density $n$.

instead of in one large single crystal (see below). Some small crystallites can still move and absorb microwave energy, which explains why we only observed a kink and not a sharp jump down to $\alpha=0$ at solidification. Similar results have been found in earlier low-frequency conductivity measurements of 2DES on solid $\mathrm{Ne},{ }^{24}$ on solid $\mathrm{H}_{2},{ }^{18}$ and on helium films, ${ }^{25}$ while previous microwave absorption studies have not detected such a transition probably because $n$ was too low $^{13}$ or because the surface quality of the polymer foil was not very good. ${ }^{14}$

Figure 3 shows three charging runs at two different temperatures and on helium films of two different initial thicknesses covering a PMMA layer approximately $25 \mathrm{~nm}$ thick. The shape of the curves, an initial linear region followed by a kink and a less steep line, is similar to that displayed in Fig. 2. In the low-density region, the curves A and B, measured at the same temperature $T=1.16 \mathrm{~K}$, overlap, which means that within the experimental resolution their relaxation time is the same, $\tau \simeq 9.4 \times 10^{-11} \mathrm{~s}$. This value is very close to the theoretical value of $\simeq 11 \times 10^{-11} \mathrm{~s}$ calculated for bulk helium and a clamping field $E_{\perp}=130 \mathrm{kV} / \mathrm{m}$, corresponding to a typical electron density of $1.5 \times 10^{9} \mathrm{~cm}^{-2}$. In other words, the PMMA substrate is sufficiently smooth and low polarizable that already on a helium film of $d_{\mathrm{He}} \geqslant 25 \mathrm{~nm}$ the SSE highfrequency mobility is practically the same as on bulk helium. Run $\mathrm{C}$, taken at $1.5 \mathrm{~K}$, has instead a relaxation time $\tau \simeq 2.6 \times 10^{-11} \mathrm{~s}$, similar to the above mentioned value measured on PFPE oil at $1.28 \mathrm{~K}$, suggesting that the PMMA substrate is probably smoother than the PFPE oil of Fig. 2. This comes to no surprise given the difficulty at spreading this oil onto the silicon platelet. 


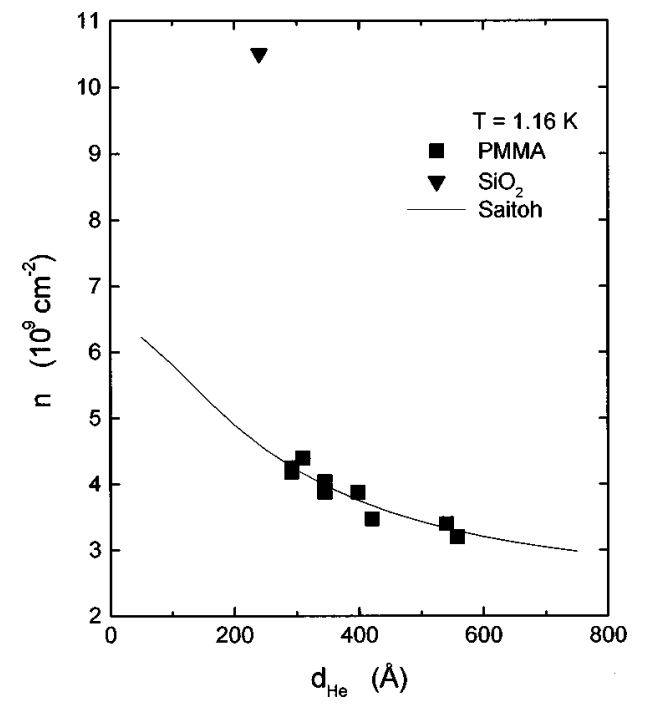

FIG. 4. Melting density $n_{M}$ of the SSE crystal on charged helium films of different thickness. The uncertainty in the absolute value of the electron density is about $15 \%$. For further information see text.

An equally interesting feature of Fig. 3 concerns the location $n_{M}$ of the kinks, which is a function of temperature and/or helium film thickness. More precisely, as $d_{\mathrm{He}}$ increases the kink moves to lower electron densities. This obviously excludes that the kink is due to localization of single electrons induced by surface defects. If this were the case, on a thick film the influence of the substrate roughness would be significantly reduced ${ }^{13}$ and this would imply that a larger fraction of electrons could move and absorb microwave radiation. In other words, we would expect an increase in $\alpha$ at larger $d_{\mathrm{He}}$ rather than the decrease observed in Fig. 3.

A consistent explanation of our observations is based on the screening of the direct Coulomb interaction caused by the presence of a dielectric surface. As Eq. (1) shows, the expected melting temperature of a 2DES crystal is an increasing function of $d_{\mathrm{He}}$. This is more clearly seen in Fig. 4, where the location $n_{M}$ of the kinks is plotted versus the equilibrium thickness of the charged helium film at a constant temperature $T=1.16 \mathrm{~K}$. For comparison we show in Fig. 4 also the prediction according to Saitoh's theory (1), calculated with $\Gamma=124$ (solid line). The agreement with our data is quite satisfactory. We should mention here that we have also made a comparison based on an interpolation formula by Peeters and Platzman, ${ }^{11}$ and found a discrepancy of about $20 \%$, which, however, can be attributed to an error in Ref. 11 as pointed out by Saitoh. ${ }^{12}$

Figure 4 also shows a point representative of our results on $\mathrm{SiO}_{2}$ (c.f. Ref. 3). This time the agreement with Saitoh's prediction of a melting density $n_{M} \approx 7 \times 10^{9} \mathrm{~cm}^{-2}$ for $\Gamma=127$ is very poor. One possible explanation of such a large discrepancy is that at the melting densities observed on $\mathrm{SiO}_{2}$ quantum corrections to Eq. (1) start to become important. Actually, the experimental point for $\mathrm{SiO}_{2}$ in Fig. 4 corresponds to a Fermi temperature $T_{F} \approx 0.3 \mathrm{~K}$.

We have also measured the dependence of the melting density with temperature in the range $1.15-1.6 \mathrm{~K}$ and on two charged helium film thicknesses of $\approx 300$ and $\approx 500 \AA$. These results are presented in Fig. 5 together with the pre-

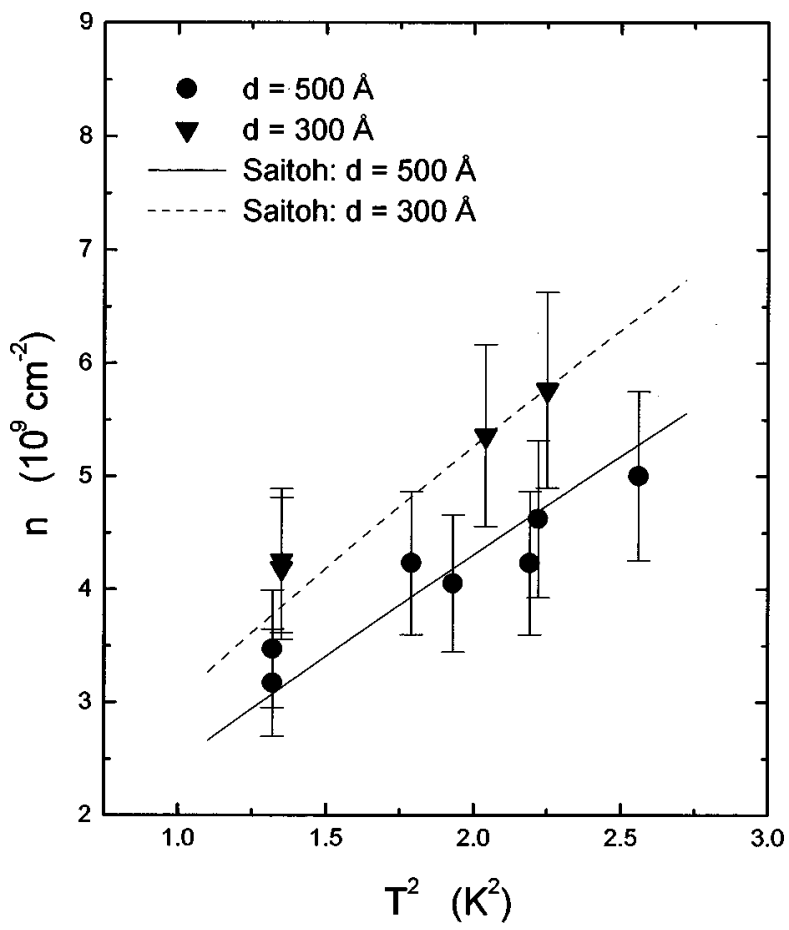

FIG. 5. Temperature dependence of the melting density $n_{M}$ of the SSE crystal. For further information see text.

dictions of Saitoh's formula for $D=300 \AA$ and $D=500 \AA$. The two lines represent the fit of the data points to Eq. (1) using $\Gamma$ as a free parameter. In this way we can get a new independent estimate of $\Gamma$. We find that the best fit to the data, see Fig. 5, is achieved with $\Gamma=117 \pm 15$, quite close to that determined from Fig. 4 and to the value of 127 found for 2DES on bulk helium. ${ }^{2,5}$ Melting transition data taken by Jiang, Stan, and Dahm ${ }^{22}$ on helium films wetting a smooth glass surface and reanalyzed by $\mathrm{Saitoh}^{12}$ also show good agreement with Eq. (1).

In our measurements we could identify the solidification point of the SSE's as a kink in the charging curves of $\chi$ and $\alpha$. However, as is shown in Fig. 6, in some runs on freshly prepared PMMA we observed a pronounced resonance structure in $\alpha(n)$ and $\chi(n)$, reminiscent of a plasma resonance, with its peak very close to the point where we expected the kink. Those resonance structures did appear only in the first few charging runs of two series of measurements taken on two fresh PMMA layers. During subsequent charging runs, the curves $\alpha(n)$ and $\chi(n)$ continuously changed their shape, finally arriving at that characteristic of Figs. 2 and 3. At that point, the data became reproducible from run to run. Although we have not yet found out how this resonance can be systematically reproduced, its characteristic shape can be well explained in terms of collective excitations of the SSE's and we attribute its occurrence to particular clean preparation conditions of the PMMA surface in these runs. As we already mentioned, firing the filament causes charging of some rough spots on the PMMA surface by electrons which have tunnelled through the helium film. These charged spots act as new scattering centers which destroy the plasma resonance over the platelet. On the other hand, they prevent further leaking of electrons through the film and this may be the reason why the final structure of Figs. 2 and 3 is rather well reproducible from run to run. 


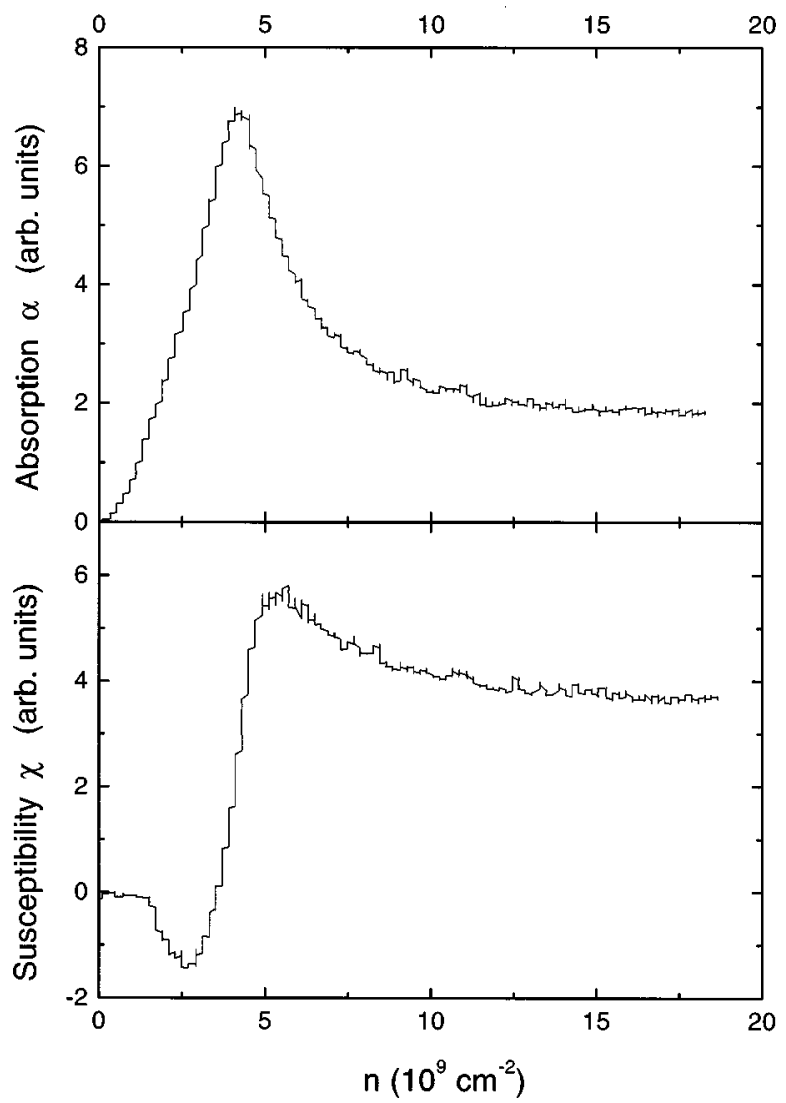

FIG. 6. Plasma resonance observed at $T=1.16 \mathrm{~K}$ on a helium film (initial thickness $\approx 340 \AA$ ) covering a freshly prepared PMMA layer. For further information see text.

Our interpretation of this observation is as follows. In the dispersion relation of longitudinal excitations of the twodimensional electron plasma $\omega\left(k_{l}\right)$ the freezing is characterized by an energy gap which opens at $k_{l}=0$. On bulk helium this is due to the phonon-ripplon coupling owing to the periodicity of the crystal, ${ }^{1,2}$ whereas on a thin helium film such a coupling is less effective due to the presence of random pinning centers. In this disordered case an energy gap still exists due to the sum of restoring forces exerted on the domains which grow with the density of electrons and with the number of pinning sites. ${ }^{26}$ (Without an energy gap the freezing of a two-dimensional electron plasma would be hardly observable in a longitudinal excitation experiment since the solidification does not affect the longitudinal eigenfrequency.) At the sharp resonance point of Fig. 6 the operating frequency $\omega \simeq 6.3 \times 10^{10} \mathrm{~s}^{-1}$ and the longitudinal wavevector $k_{l}=157 \mathrm{~m}^{-1}$, given by the cavity length $20 \mathrm{~mm}$, have met the dispersion relation of the plasma. Since at this density
( $n \simeq 4 \times 10^{9} \mathrm{~cm}^{-2}$ ) the longitudinal plasma resonance of the free SSE's, $f_{P}^{2}=n e^{2} k / 2 \pi m,{ }^{27}$ is around $0.5 \mathrm{GHz}$, it follows that the effect comes from the energy gap which has opened due to solidification. We do not know whether this energy gap is due to the optical phonon-ripplon coupled mode or, more likely, to local vibration-modes of pinned domains, but since the resonance is so sharp it represents in any case the "cleanest" state of this system. The continuous evolution from a resonance to a kink in subsequent charging curves shows that the shape shown in Figs. 2 and 3 represents a disordered state. Their characteristic feature, the kink as a residue from a sharp resonance, reflects the broad distribution of possible collective excitations in the system.

\section{CONCLUSIONS}

The dielectric response of a 2DES on helium films adsorbed on polymer substrates has been measured by means of a $10 \mathrm{GHz}$ microwave cavity. The simultaneous measurement of the real and imaginary parts of $\epsilon(k, \omega)$ allows for a straightforward determination of the freezing transition of the SSE's. The effect of the screening of the interparticle Coulomb interaction caused by the presence of the dielectric substrate on such a 2DES transition has been studied at different temperatures and various helium film thicknesses. Quite satisfactory quantitative agreement has been found between our data on PMMA and Saitoh's formula based on the Kosterlitz-Thouless melting criterion.

The low polarizability and simplicity in the preparation of a PMMA layer with a smooth surface and a thickness which is possible to tune in a relatively ample range make this polymer an ideal substrate for the investigation of charged helium films. However, its low permittivity reduces the shrinking of the 2DES crystal phase caused by substrate screening. As a consequence, on PMMA we have not seen any indication of a quantum melting of the crystal up to the maximum densities achieved in our measurements, $n \leqslant 2 \times 10^{11} \mathrm{~cm}^{-2}$. From this point of view, the use of a more polarizable insulating layer such as $\mathrm{SiO}_{2}$ is preferable, although its preparation is much more delicate and the effects of surface defects and charge localization are more important and pronounced than on PMMA.

\section{ACKNOWLEDGMENTS}

We are grateful to B. Bitnar, M. Cole, K. Dransfeld, F. Penning, V. Shikin, and especially O. Tress for many useful and clarifying discussions. The PMMA layers have been kindly prepared by Kevin Phelan and Martin Boltau of Professor Mlynek's group at Konstanz University. This research has been partially supported by the HCM-network of the European Community, contract No. ERBCHRXCT90374.
${ }^{1}$ See, e.g., 2D Electron Systems on Helium and Other Cryogenic Substrates, edited. by E. Y. Andrei (Kluwer, Dordrecht, 1997).

${ }^{2}$ C. C. Grimes and G. Adams, Phys. Rev. Lett. 42, 795 (1979); D. S. Fisher, B. I. Halperin, and P. M. Platzman, ibid. 42, 798 (1979).
${ }^{3}$ G. Mistura, T. Günzler, B. Bitnar, S. Neser, and P. Leiderer, Surf. Sci. 361/362, 831 (1996).

${ }^{4}$ E. Wigner, Phys. Rev. 46, 1002 (1934).

${ }^{5}$ G. Deville, J. Low Temp. Phys. 72, 135 (1988).

${ }^{6}$ F. M. Peeters, in Physics of the Two-dimensional Electron Gas, 
edited by J. T. Devreese and F. M. Peeters, Vol. 157 of NATO Advanced Study Institute Series B: Physics (Plenum Press, New York 1987), p. 393.

${ }^{7}$ Yu. P. Monarkha and V. B. Shikin, Fiz. Nizk. Temp. 8, 563 (1982) [ Sov. J. Low Temp. Phys. 8, 279 (1982)].

${ }^{8}$ H. Etz, W. Gombert, W. Idstein, and P. Leiderer, Phys. Rev. Lett. 53, 2567 (1984).

${ }^{9}$ P. M. Platzman and H. Fukuyama, Phys. Rev. B 10, 3150 (1974).

${ }^{10}$ R. H. Morf, Phys. Rev. Lett. 43, 931 (1979).

${ }^{11}$ F. M. Peeters and P. M. Platzman, Phys. Rev. Lett. 50, 2021 (1983); F. M. Peeters, Phys. Rev. B 30, 159 (1984).

${ }^{12}$ M. Saitoh, Phys. Rev. B 40, 810 (1989).

${ }^{13}$ Yu. Z. Kovdrya, F. F. Mende, and V. A. Nikolaenko, Sov. J. Low Temp. Phys. 10, 589 (1984); F. F. Mende, Yu. K. Kovdrya, and V. A. Nikolaenko, ibid. 11, 355 (1985).

${ }^{14}$ B. Lehndorff and K. Dransfeld, J. Phys. (France) 50, 2579 (1989).

${ }^{15}$ T. Günzler; Ph.D. thesis, Konstanz University, 1994.

${ }^{16}$ In principle, retardation effects should be taken into account for films thicker than $\approx 400 \AA$. Since the main uncertainty arises from the lack of a precise $\alpha$ value for PMMA and because of the thinning of the film due to the pressure excerted by the electrons supported by the helium film, it is sufficient to use the formula for the unretarded case.

${ }^{17}$ E. Cheng and M. W. Cole, Phys. Rev. B 38, 987 (1988); Milton Cole (private communication).

${ }^{18}$ F. Mugele and P. Leiderer, J. Phys. IV 3, 45 (1993).

${ }^{19}$ G. Mistura, T. Günzler, and P. Leiderer (unpublished).

${ }^{20}$ V. B. Shikin and Yu P. Monarkha, Sov. J. Low Temp. Phys. 1, 459 (1975).

${ }^{21}$ M. Saitoh, J. Phys. Soc. Jpn. 42, 201 (1977).

${ }^{22}$ H. W. Jiang, M. A. Stan, and A. J. Dahm, Surf. Sci. 196, 1 (1988); H. W. Jiang and A. J. Dahm, ibid. 229, 352 (1990).

${ }^{23}$ K. Kono, U. Albrecht, and P. Leiderer, J. Low Temp. Phys. 82, 279 (1991); K. Kono, U. Albrecht, and P. Leiderer, ibid. 82, 423 (1991).

${ }^{24}$ K. Kajita, Surf. Sci. 142, 86 (1984); K. Kajita, J. Phys. Soc. Jpn. 54, 4092 (1985).

${ }^{25}$ H. W. Jiang and A. J. Dahm, Phys. Rev. Lett. 62, 1396 (1989).

${ }^{26}$ A. G. Eguiluz, A. A. Maradudin, and R. J. Elliott, Surf. Sci. 113, 426 (1982).

${ }^{27}$ C. C. Grimes and G. Adams, Phys. Rev. Lett. 42, 795 (1979). 